\title{
A CLASS OF TWO-BRIDGE KNOTS WITH PROPERTY-P
}

\author{
E. J. MAYLAND, JR. ${ }^{1}$
}

\begin{abstract}
A knot $k$ has property-P provided no simply connected manifold results from performing a nontrivial elementary surgery along $k$. We establish property-P for certain families of two-bridge knots generalizing twist knots (Whitehead doubles of the trivial knot).
\end{abstract}

Hamstrom and Jerrard [3] associate a two-bridge knot or link to each ordered pair of relatively prime integers $(\alpha, \beta)$ with $|\alpha|>|\beta|$. For each $p=1,2, \ldots$ we will consider the family of two-bridge knots associated with the pairs $(|2 p(2 m-1)+1|, 2 p), \quad m= \pm 1, \pm 2, \ldots$ Schubert [10] had earlier used ordered pairs of odd, relatively prime integers to classify the associated knots, and in these terms we will consider the knots $(4 p m-2 p+$ $1,4 p(m-1)+1)$, for $m>0, p>0$ and $(-4 p m+2 p-1,-4 p m-1)$ when $m<0$ and $p>0$. A knot $k$ has property-P provided no simply connected manifold results from performing a nontrivial elementary surgery along $k$ (see, e.g., [1]). We show that each knot in the union of the above families has property-P. This generalizes the result of Bing and Martin [1], González-Acuña [2], and, more recently, Riley [9], each of whom established the case $p=1$ (yielding the family of twist-knots, or Whitehead doubles of the trivial knot). The class of knots we consider is indicated in Figure 1, and we remark that our result also extends that of Neuzil [7], who showed that when a regular neighborhood of a nontrivial knot $J$ is replaced by the solid torus $T$ of the figure, the image of $K$ is a knot with property-P.

To begin the proof, we observe that the group of a two-bridge knot $(\alpha, \beta)$ can be computed directly from the characterising integers following [6] and [8].

We consider first the case $m>0$. Taking the equivalent knots $(4 p m-2 p$ $+1,4 p(m-1)+1)$, we find

$$
\begin{aligned}
G=\pi_{1}\left(S^{3}-k\right) & =\left\langle x_{1}, x_{2} \mid W x_{1} W^{-1}=x_{2}\right\rangle \\
\text { where } W & =\left(\left(x_{1}^{-1} x_{2}\right)^{m-1} x_{1}^{-1}\left(x_{2}^{-1} x_{1}\right)^{m-1} x_{2}^{-1}\right)^{p} \text { and } m, p>0 .
\end{aligned}
$$

Presented to the Society, January 22, 1976; received by the editors September 11, 1975 and, in revised form, July 12, 1976.

AMS (MOS) subject classifications (1970). Primary 55A05, 55A25, 55A40, 57A10.

Key words and phrases. Fundamental group, 3-manifold, knots, property-P.

${ }^{1}$ Partially supported by National Research Council of Canada grant \#A8207.

- American Mathematical Society 1977 
In this group we will take $x_{1}$ and

$$
L=x_{1}^{-4 p} \cdot W^{-1} \cdot\left(\left(x_{1} x_{2}^{-1}\right)^{m-1} x_{1}\left(x_{2} x_{1}^{-1}\right)^{m-1} x_{2}\right)^{p}
$$

as a (meridian, null-homologous longitude) pair on the boundary of a regular neighborhood of $k$. If we perform an elementary surgery along $k$ by replacing this regular neighborhood by a solid torus $T$ from $S^{3}$ in such a way that the meridian for $T$ is identified with a simple closed curve in the class of $L^{n} x_{1}^{n_{1}}$, then the group $G\left(k: n_{1}, n\right)$ of the resulting manifold is obtained by adding the relation $L^{n} x_{1}^{n_{1}}=1$ to the group (1). If $\left(n_{1}, n\right) \neq( \pm 1,0)$, we say the surgery was nontrivial, and we will now show that the group $G\left(k: n_{1}, n\right)$ is nontrivial for all nontrivial surgeries.

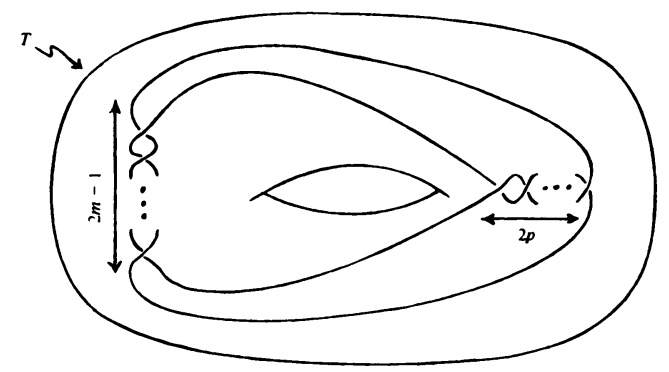

Figure 1a.

The two-bridge knots $(2 p(2 m-1)+1,2 p)=(4 p m-2 p+1,4 p m-4 p+1)$ with $m>0$ and $p>0$.

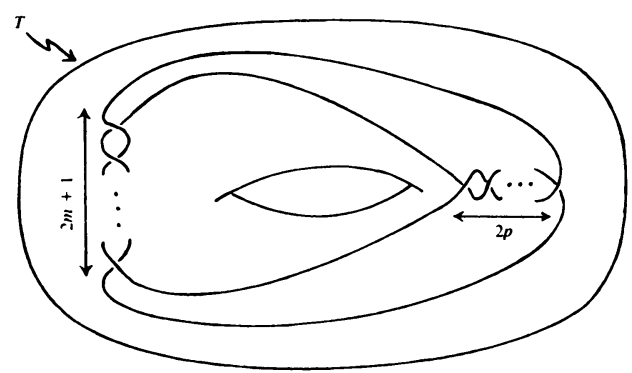

Figure 1b.

The two-bridge knots $(|2 p(2 m-1)+1|, 2 p)=(-4 p m+2 p-1,-4 p m-1)$ with $m<0$ and $p>0$.

By homology considerations $G\left(k: n_{1}, n\right) \neq 1$ if $n_{1} \neq \pm 1$. Thus by reversing orientations, if necessary, we can restrict ourselves to the case $n_{1}=+1$. In $G(k: 1, n)$, we define $w=x_{1}^{-1} x_{2}$ and delete $x_{2}=x_{1} w$, yielding $G(k: 1, n)=\left\langle x_{1}, w\right|\left(w^{m-1} x_{1}^{-1} w^{-m} x_{1}^{-1}\right)^{p} \cdot x_{1} \cdot\left(w^{m-1} x_{1}^{-1} w^{-m} x_{1}^{-1}\right)^{-p}=x_{1} w$,

$$
\left.\left[x_{1}^{-4 p}\left(x_{1} w^{m} x_{1} w^{-m+1}\right)^{p} \cdot\left(x_{1} w^{-m+1} x_{1} w^{m}\right)^{p}\right]^{n} x_{1}=1\right\rangle
$$


Defining $k=x_{1} w^{m}$ and deleting $x_{1}=k w^{-m}$ yields

$$
\begin{aligned}
& G(k: 1, n)=\langle k, w|\left(w^{2 m-1} k^{-2}\right)^{p} \cdot k w^{-m} \cdot\left(w^{2 m-1} k^{-2}\right)^{-p}=k w^{-m+1}, \\
& {\left.\left[\left(k w^{-m}\right)^{-4 p} \cdot\left(k^{2} w^{-2 m+1}\right)^{p} \cdot\left(k w^{-2 m+1} k\right)^{p}\right]^{n} k w^{-m}=1\right\rangle . }
\end{aligned}
$$

To this we adjoin the relation $w^{2 m-1}=1$, so that $G(k: 1, n)$ is generated by $k$ and $w^{m}$. Then, using the fact that $L$ and $x_{1}$ lie on a common torus and must therefore have commuting images in any homomorphic image of the knot group, we send $w^{-m}$ and $k$ to $A$ and $B$, mapping this quotient onto

$$
\bar{G}_{n}=\left\langle A, B \mid 1=A^{2 m-1}=B^{4 p}=(A B)^{4 p n-1}=\left(A B^{2 p}\right)^{2}\right\rangle
$$

where $p, m>0$ and $n \neq 0$.

We treat the case $m<0$ analogously, temporarily replacing $m$ by its absolute value. The two-bridge knots considered are therefore of the form $(4 p m+2 p-1,2 p)$ with $p>0$ and $m>0$. The groups of the equivalent knots $(4 p m+2 p-1,4 p m-1)$ are given by

$$
G=\pi_{1}\left(s^{3}-k\right)=\left\langle x_{1}, x_{2} \mid W x_{1} W^{-1}=x_{2}\right\rangle
$$

where $W=x_{2}^{-1}\left(x_{2}\left(x_{1}^{-1} x_{2}\right)^{m} x_{1}\left(x_{2}^{-1} x_{1}\right)^{m}\right)^{p} x_{1}^{-1}$ and $m, p>0$.

After the corresponding sequence of Tietze transformations, we map the appropriate quotient of this group homomorphically onto the group

$$
\bar{G}_{n}=\left\langle A, B \mid 1=A^{2 m+1}=B^{4 p}=(A B)^{4 p n+1}=\left(A B^{2 p}\right)^{2}\right\rangle
$$

where $p, m>0$ and $n \neq 0$.

Apparently the presentations (4) and (4') are identical if we allow positive and negative (nonzero) values of $m$, which amounts to abandoning our temporary convention of writing $m$ for its absolute value in the (prime) case $m<0$. It thus suffices for both cases to show the groups $\bar{G}_{n}$ of (4) are nontrivial for $p>0$ and $m, n \neq 0$.

We have already remarked that the case $p=1$ was settled by Bing and Martin, etc., and observe that the value $m=1$ yields the $K(2 p+1,2)$ torus knot with two-bridge form $(2 p+1,1)$. Torus knots were shown to have property-P by Hempel [4].

We map (4) to the following group isomorphically by defining $C=A B^{2 p}$ and deleting $A$ :

$$
\bar{G}_{n}=\left\langle B, C \mid 1=B^{4 p}=C^{2}=\left(C B^{-2 p+1}\right)^{4 p n-1}=\left(C B^{2 p}\right)^{2 m-1}\right\rangle
$$

We consider this as the result of adjoining the relations $\left(C B^{-2 p+1}\right)^{4 p n-1}=$ 1 and $\left(C B^{2 p}\right)^{2 m-1}=1$ to the free product of cyclic groups of orders 2 and $4 p$. Applying the theory of small cancellation over free products (see, e.g., [5]), we find the relations satisfy both triangle conditions and the small cancellation 
condition $C(d)$ for $d=\min \{|4 p n-1|,|2 m-1|\}$. Since $p>1$ and $m \neq 0,1$, this shows all the groups (5) are nontrivial unless $|2 m-1|=3$.

The remaining case is

$$
\bar{G}_{n}=\left\langle B, C \mid 1=B^{4 p}=C^{2}=\left(C B^{2 p}\right)^{3}=\left(C B^{-2 p+1}\right)^{4 p n-1}\right\rangle
$$

where $p>1$ and $n \neq 0$.

This group is generated by $B^{2 p-1}$ and $C$, and we can therefore present it as

$$
\bar{G}_{n}=\left\langle C, D \mid 1=C^{2}=D^{4 p}=\left(C D^{2 p}\right)^{3}=(C D)^{4 p n-1}\right\rangle
$$

where $p>1$ and $n \neq 0$.

We will show these groups are nontrivial using permutation representations related to those of González-Acuña [2].

If $n=+1$, we map $\left\langle C, D \mid 1=C^{2}=D^{4 p}=\left(C D^{2 p}\right)^{3}=(C D)^{4 p-1}\right\rangle$ into the alternating group $A_{8 p-2}$ via the homomorphism $\theta_{1}$ determined by

$$
\begin{aligned}
C & \mapsto(1,4 p+1)(2,6 p+1)(3,6 p+2) \cdots(2 p-1,8 p-2)(2 p, 6 p-1), \\
D & \mapsto(1,2, \ldots, 4 p)(4 p+1,4 p+2, \ldots, 6 p) .
\end{aligned}
$$

If $n>+1$, we observe the homomorphism $\theta_{2}$ sending $\langle c, d| 1=c^{2}=d^{4 p}$ $=\left(c d^{2 p}\right)^{3}=(c d)^{4 p}>$ into $A_{8 p}$ via

$$
\begin{aligned}
& C \mapsto(1,4 p+1)(2,6 p+2)(3,6 p+3) \cdots(2 p-1,8 p-1)(2 p, 6 p), \\
& D \mapsto(1,2, \ldots, 4 p)(4 p+1, \ldots, 6 p)(6 p+1,6 p+2)(8 p-1,8 p) .
\end{aligned}
$$

Then the above two maps can be combined to provide a homomorphism from (7) into the alternating group $A_{8 p n-2}$ via

$$
\begin{gathered}
C \mapsto \prod_{i=1}^{n-1}[(8 p i-8 p+1,8 p i-4 p+1)(8 p i-8 p+2,8 p i-2 p+2) \\
\ldots(8 p i-6 p-1,8 p i-1)(8 p i-6 p, 8 p i-2 p)] \\
\quad \times(8 p n-8 p+1,8 p n-4 p+1)(8 p n-8 p+2,8 p n-2 p+1) \\
\ldots(8 p n-6 p-1,8 p n-2)(8 p n-6 p, 8 p n-2 p-1), \\
\mapsto \prod_{i=1}^{n}[(8 p i-8 p+1, \ldots, 8 p i-4 p)] \\
\quad \times \prod_{i=1}^{n-1}[(8 p i-2 p+1,8 p i-2 p+2)(8 p i-1,8 p i) \\
\quad \times(8 p i-4 p+1,8 p i+4 p+2, \ldots, 8 p i+6 p)] \\
\quad \times(8 p n-4 p+1,4 p+2, \ldots, 6 p) .
\end{gathered}
$$

The case $n<0$ is completely analogous. If $n=-1$ we map the group 
$\left\langle C, D \mid 1=C^{2}=D^{4 p}=\left(C D^{2 p}\right)^{3}=(C D)^{4 p+1}\right\rangle$ into the alternating group $A_{8 p+2}$ via a homomorphism $\theta_{3}$ defined by

$$
\begin{aligned}
& C \mapsto(1,4 p+1)(2,6 p+2)(3,6 p+3) \\
& \ldots(2 p-2,8 p-2)(8 p+2,4 p-1)(2 p, 4 p), \\
& D \mapsto(1, \ldots, 4 p)(4 p+1, \ldots, 6 p)(6 p+1, \ldots, 8 p)(8 p+1,8 p+2) .
\end{aligned}
$$

If $n<-1$, the homomorphisms $\theta_{3}$ and $\theta_{2}$ are combined just as in the case $n>+1$. This shows the nontriviality of the groups (5) and completes the proof.

\section{REFERENCES}

1. R. H. Bing and J. M. Martin, Cubes with knotted holes, Trans. Amer. Math. Soc. 155 (1971), 217-231. MR 43 \#4018a.

2. F. González-Acuña, Dehn's construction on knots, Bol. Soc. Mat. Mexicana (2) 15 (1970), 58-79. MR 50 \#8495.

3. M. E. Hamstrom and R. P. Jerrard, Collapsing a triangulation of a "knotted" cell, Proc. Amer. Math. Soc. 21 (1969), 327-331. MR 39 \# 4831.

4. John Hempel, $A$ simply connected 3-manifold is $S^{3}$ if it is the sum of a solid torus and the complement of a torus knot, Proc. Amer. Math. Soc. 15 (1964), 154-158. MR 28 \#599.

5. R. Lyndon, On Dehn's algorithm, Math. Ann. 166 (1966), 208-228. MR 35 \#5499.

6. K. Murasugi, Remarks on knots with two bridges, Proc. Japan Acad. 37 (1961), $294-297$. MR 25 \#2599.

7. J. P. Neuzil, Surgery on a curve in a solid torus, Trans. Amer. Math. Soc. 204 (1975), 385-406. MR 51 \#4212.

8. R. Riley, Parabolic representations of knot groups. I, Proc. London Math. Soc. (3) 24 (1972), 217-242. MR 45 \#9313.

9. __ Knots with parabolic property-P, Quart. J. Math. (2) 25 (1974), 273-283.

10. H. Schubert, Knoten mit zwei Brücken, Math. Z. 65 (1956), 133-170. MR 18, 498.

Department of Mathematics, York University, Downsview, Ontario M3J 1P3, Canada 